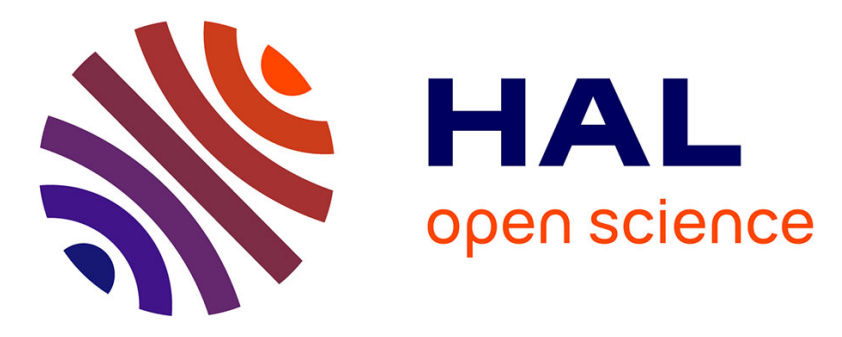

\title{
A Fluid Approach for Performance Analysis of LTE-A Networks with Relays
}

Mattia Minelli, Maode Ma, Jean-Marc Kélif, Marceau Coupechoux, Marc Sigelle

\section{- To cite this version:}

Mattia Minelli, Maode Ma, Jean-Marc Kélif, Marceau Coupechoux, Marc Sigelle. A Fluid Approach for Performance Analysis of LTE-A Networks with Relays. IEEE International Symposium on Personal, Indoor and Mobile Radiocommunications (PIMRC), Workshop on Cooperative and Heterogeneous Cellular Networks, Sep 2013, London, United Kingdom. pp.1-5. hal-00844869

\section{HAL Id: hal-00844869 \\ https://hal-imt.archives-ouvertes.fr/hal-00844869}

Submitted on 16 Jul 2013

HAL is a multi-disciplinary open access archive for the deposit and dissemination of scientific research documents, whether they are published or not. The documents may come from teaching and research institutions in France or abroad, or from public or private research centers.
L'archive ouverte pluridisciplinaire HAL, est destinée au dépôt et à la diffusion de documents scientifiques de niveau recherche, publiés ou non, émanant des établissements d'enseignement et de recherche français ou étrangers, des laboratoires publics ou privés. 


\section{A Fluid Approach for Performance Analysis of LTE-A Networks with Relays}

\author{
Mattia Minelli ${ }^{1}$, Maode $\mathrm{Ma}^{2}$
}

\author{
Jean-Marc Kelif ${ }^{3}$, Marceau Coupechoux ${ }^{4}$ \\ Marc Sigelle ${ }^{5}$
}

\begin{abstract}
In this paper, we address the problem of the impact of relays on the performance of a LTE-A based network. In this aim, we propose a new framework, the "fluid model for LTE-A networks with relays". We derive from this model analytical simple formulas for the Signal to Interference plus Noise ratio (SINR), taking into account inter-cell interference, which allow to study performance of wireless networks constituted by macro base stations and relays. In our analysis, a fraction $\tau$ of radio resources is dedicated to the base-station (eNodeB)/relay nodes (RN) communication. By using the fluid model, we instantaneously quantify an optimized value of $\tau$, according to the number of relays, which allows to increase the capacity. In the remaining resources, eNodes-B and RNs transmit simultaneously to users. During this phase, the network is densified in the sense that the transmitters density and so network capacity are increased. Performance results show that cell capacity is boosted thanks to densification despite a degradation of the signal quality.
\end{abstract}

\section{INTRODUCTION}

Relaying is a promising feature of future cellular networks. The scenarios envisioned by the two standards IEEE $802.16 \mathrm{j}$ (for WiMAX networks) and 3GPP Release 10 and 11 (LTEAdvanced) are the following: (a) coverage extension (including indoor coverage and shadowed zone mitigation), (b) capacity boost, (c) group mobility. In this paper, we tackle the problem of relay impact on the performance of a LTE-A network in terms of Signal to Interference plus Noise Ratio (SINR) and cell capacity. To this aim, we propose a new framework, called fluid model for relay-based cellular networks, that allows quick calculations of the SINR.

There is a large literature on the performance evaluation of relay-based cellular networks. For example, [1] provides a comparative study between repeaters and relays based on system level simulations for the computation of the SINR. It is shown that is it preferable in terms of cell throughput that base stations and relays transmit simultaneously, rather than on orthogonal resources. 3GPP has also conducted extensive simulations for assessing the gains provided by relays [2].

\footnotetext{
${ }^{1}$ M. Minelli is with Telecom ParisTech, France and Nanyang Technological University, Singapore. Email: mattia.minelli@telecom-paristech.fr

${ }^{2}$ M. Ma is with Nanyang Technological University, Singapore Email: emdma@ntu.edu.sg

3 Jean-Marc Kelif is with Orange Labs, France. jeanmarc.kelif@orange.com Email: jeanmarc.kelif@ orange.com

${ }^{4}$ M. Coupechoux is with Telecom ParisTech, France. Email: marceau.coupechoux@telecom-paristech.fr

${ }^{5}$ M. Sigelle is with Telecom ParisTech, France. Email: marc.sigelle@telecom-paristech.fr
}

Several papers propose dynamic resource allocation schemes for relay-based cellular networks making use of SINR calculations based on simulations, e.g. [3]. There are also optimization techniques for relay placements, which rely on extensive SINR computations. For example, in [4], authors propose a method for optimizing the relay placement using a Genetic algorithm with the aim of maximizing system spectral efficiency. Results are presented in terms of optimal placement and distributions of throughputs and spectral efficiency. Every iteration of the proposed genetic algorithm includes a simulation for the SINR and the spectral efficiency computation. This can lead to cumbersome computation times.

In order to avoid this drawback, some papers propose SINR-based analytical studies, like [5], [6], [7]. They however assume very simple models, where only the closest interferer is taken into account. Although they provide interesting insights on the relay deployment, such models can lead to inaccurate performance evaluations. For this reason, we propose in this paper a simple analytical expression of the SINR based on a fluid model of the network, which can speed up numerical calculations, without meaningfully affecting the accuracy of the SINR evaluation.

The organization of the paper is as follows. In Section II, we present the network model. In Section III, we derive SINR formulas. In Section IV, we propose a fluid model for cellular networks with relays and we validate the approach with Monte Carlo simulations. Examples of performance results are provided in Section $\mathrm{V}$ and the last section concludes the paper.

\section{Network Model}

In this section, we describe the considered network topology, the frames structure and the channel model.

\section{A. Network Topology}

We consider a single frequency cellular LTE-A based network consisting of omnidirectional eNodes-B (eNB) hexagonal cells. Let $R_{c}$ be the half-distance between two neighbor eNBs and $\rho_{e N B}$ the eNB density. eNBs transmit at power $P$.

In each cell, $n$ RNs are deployed with a regular pattern and controlled by the eNB. We focus on capacity evaluation for the downlink. The generic relay deployment is illustrated in Fig. 1: relays are regularly deployed at distance $R_{R}$ from the eNB and at angles $\varphi+2 \pi / n$, where $\varphi$ is an offset. Note that the deployment pattern is identical in all cells ( $\varphi$ and $R_{R}$ are constant across the cells). The RN density is $\rho_{R}=n \rho_{e N B}$. RNs transmit at power $P_{R}$. 
RNs in a cell are labeled from 1 to $n$. Cells are labeled from 0 to $B$ so that a RN can be uniquely identified by $(i, k)$, where $i$ is the relay number and $k$ is the cell number. A relay $(i, k)$ is said to be of type $i$. The set of type $i$ relays form a regular pattern, where the minimum half-distance between nodes is $R_{c}$ and whose density is $\rho_{e N B}$.

We denote $r_{b}$ the distance between eNB $b$ and the UE of interest and, for simplicity, we set $r=r_{0}$. We denote $r_{i, k}$ the distance between the relay $(i, k)$ and the UE. Let also $r_{i, k^{*}(i)}=\min _{k} r_{i, k}$ be the minimum distance between the UE and a type $i \mathrm{RN}$.

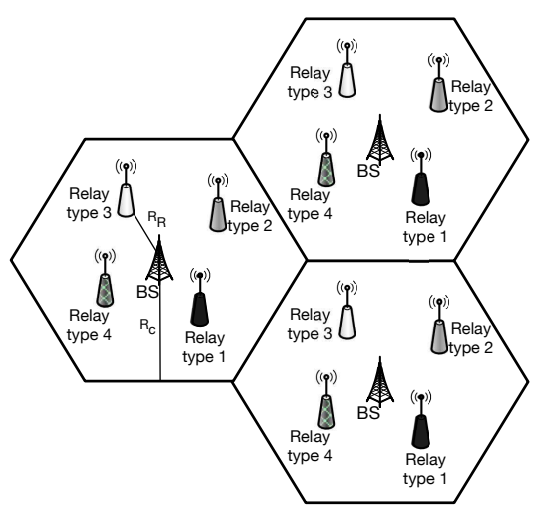

Fig. 1. Relay Deployment.

\section{B. Resource Organization}

We consider in-band half-duplex relays ${ }^{1}$. We assume a time division access between eNBs and RNs and we focus on a single frame of duration $t_{f r}=1$ (unit of time). The eNB transmits data to the relays over the Backhaul Link (BL) during a time $\tau$ and the eNB and the relays simultaneously transmit data to their respective attached UEs during $1-\tau$, respectively over the Direct Link (DL, eNB-UE link) and the Relay Link (RL, RN-UE link). We assume that UEs are served by the station from which they receives the highest pilot power (best server policy).

\section{SINR EVALUATION}

In this section, we evaluate the Signal to Interference plus Noise Ratio (SINR), $\gamma(r)$, experienced by a UE located at distance $r$ from the central eNB. We first assume that the UE is served on the DL by the eNB, then on the RL by a type $j \mathrm{RN}$. Formulas are then simplified in the next section using the fluid model approach. Let $g$ and $g_{R}$ be the path-gains on the DL and the RL resp., and $N_{t h}$ the thermal noise power.

\footnotetext{
${ }^{1}$ The study can be extended to out of band full duplex relays, the following SINR evaluation is only slightly different.
}

\section{A. UE Served by the eNode-B}

If the UE is attached to the eNB, it is interfered by all the other eNBs and all the RNs of the network, we can thus write:

$$
\begin{aligned}
\gamma(r) & =\frac{P g(r)}{\sum_{b=1}^{B} P g\left(r_{b}\right)+\sum_{k=0}^{B} \sum_{i=1}^{n} P_{R} g_{R}\left(r_{i, k}\right)+N_{t h}} \\
& =\frac{\frac{P g(r)}{\sum_{b=1}^{B} P g\left(r_{b}\right)}}{1+\sum_{i=1}^{n} \sum_{k=0}^{B} \frac{P_{R} g_{R}\left(r_{i, k}\right)}{\sum_{b=1}^{B} P g\left(r_{b}\right)}+\frac{N_{t h}}{\sum_{b=1}^{B} P g\left(r_{b}\right)}} \\
& =\frac{\gamma_{0}}{1+I_{1}+I_{2}},
\end{aligned}
$$

where

$$
\gamma_{0}=\frac{P g(r)}{\sum_{b=1}^{B} P g\left(r_{b}\right)},
$$

$$
\begin{aligned}
I_{1}= & \frac{\sum_{i=1}^{n} \sum_{k=0}^{B} P_{R} g_{R}\left(r_{i, k}\right)}{\sum_{b=1}^{B} P g\left(r_{b}\right)} \\
= & \frac{\sum_{i=1}^{n}\left(\sum_{k \neq k^{*}(i)} P_{R} g_{R}\left(r_{i, k}\right)+P_{R} g_{R}\left(r_{i, k^{*}(i)}\right)\right)}{\sum_{i=1}^{B} \Omega_{i}\left(1+\gamma_{i, k^{*}(i)}\right),} \\
& \gamma_{i, k^{*}(i)}=\frac{P_{R} g_{R}\left(r_{i, k^{*}(i)}\right)}{\sum_{b=1}^{B} P_{R} g_{R}\left(r_{i, k}\right)} \\
\Omega_{i}=\frac{N_{t h}}{\sum_{k=0, k \neq k^{*}(i)}^{B} P g\left(r_{b}\right)} & \frac{\sum_{b=1}^{B} P_{R} g_{R}\left(r_{i, k}\right)}{I_{b=1}(i)}
\end{aligned}
$$

Parameter $\gamma_{0}$ can be interpreted as the Signal to Interference Ratio (SIR) of the UE if it were served by the eNB 0 and 
if there were only eNBs in the network (i.e., RNs were not transmitting). In the same way, $\gamma_{i, k}$ can interpreted as the SIR of the UE if it were attached to relay $(i, k)$ and if there were only $i$-type relays in the network (i.e., eNBs and other types of RNs were inactive). $\Omega_{i}$ is the ratio of interference received by type $i$ relays and eNBs. For the considered UE, relay $\left(i, k^{*}(i)\right)$ is the closest of type $i$. Due to the network topology, it is possible that this relay is not controlled by the central eNB, so that $k^{*}(i)$ may be different from 0 .

\section{B. UE Served by a Relay Node}

If the UE is attached to a relay of type $j, \mathrm{UE}$ is interfered by all the eNBs and all the other RNs of the network, we can thus write:

$$
\begin{gathered}
\gamma\left(r_{j, k^{*}(j)}\right)= \\
\frac{P_{R} g_{R}\left(r_{j, k^{*}(j)}\right)}{\sum_{b=0}^{B} P g\left(r_{b}\right)+\sum_{\substack{(i, k) \neq\left(j, k^{*}(j)\right) \\
\gamma_{j, k^{*}(j)}}} P_{R} g_{R}\left(r_{i, k}\right)+N_{t h}} \\
=\frac{1+\frac{1+\gamma_{0}}{\Omega_{j}}+\sum_{i \neq j}\left(1+\gamma_{i, k^{*}(i)}\right) \Omega_{i, j}+I_{3}}{1},
\end{gathered}
$$

where

$$
\begin{aligned}
\Omega_{i, j} & =\frac{\sum_{k \neq k^{*}(i)} P_{R} g_{R}\left(r_{i, k}\right)}{\sum_{k \neq k^{*}(j)} P_{R} g_{R}\left(r_{j, k}\right)}, \\
I_{3} & =\frac{N_{t h}}{\sum_{k \neq k^{*}(j)} P_{R} g_{R}\left(r_{j, k}\right)} .
\end{aligned}
$$

Parameter $\Omega_{i, j}$ is the ratio of interference received by type $i$ relays and type $j$ relays.

\section{Fluid Model for Relay-BASEd Cellular NETWORKS}

\section{A. Recalls on the Fluid Model}

The fluid model [8] is a powerful tool for simplifying SINR formulas in a wireless network. Consider a network only constituted of regularly spaced eNBs with half inter-site distance equal to $R_{c}$, with density $\rho_{e N B}$ and transmitting at the same power $P$. Let $g(r)=K r^{-\eta}$ be the path-gain at distance $r$. Assume that a UE is at distance $r$ from its serving eNB. The total interference received by the UE can then be approximated by:

$$
\sum_{b=1}^{B} P g\left(r_{b}\right)=\frac{2 \pi \rho_{e N B} P K\left(2 R_{c}-r\right)^{2-\eta}}{\eta-2} .
$$

We refer the reader to [9] for the detailed explanation and validation through Monte Carlo simulations. The main idea is to replace a discrete set of transmitters by a continuum and thus transform discrete sums into integrals. Beside its simplicity, the main advantage of this approach is to obtain a function that only depends on the distance to the serving eNB rather than on all the distances to every interferer.

\section{B. Extension to Relay-Based Networks}

We now extend this concept to a relay-based cellular LTE-A network. Contrary to what is assumed in [8], such a network shows inhomogeneities: inter-distance between neighboring stations is not constant and eNB and $\mathrm{RN}$ transmit powers are different.

The network considered in this paper can however be seen as constituted of one regular subnetwork of eNBs and $n$ regular subnetworks of relays. These last ones generate an interfering power expressed as $\sum_{k=0}^{B} \sum_{i=1}^{n} P_{R} g_{R}\left(r_{i, k}\right)$ (see (1)). Fig 1 shows an example of a relay-based network with 4 RNs per cell. It can be observed that the set of each type of relays constitutes a regular subnetwork. The half-inter-relay distance of this subnetwork is $R_{c}$, the same as the eNB subnetwork. As a consequence, the fluid model can be used for computing the interference generated by this subnetwork. The fluid model can thus be used for computing the interference generated by each subnetwork of RNs and eNBs. This is the basic idea of the extension of the fluid model to a cellular network with RNs.

As a consequence, in our study, the fluid model can be used for computing the interference received from all eNBs of the network on the one hand and from each type $i(i \in\{1, \ldots, n\})$ relays (i.e. subnetwork) on the other hand, taking into account all relays of the network.

Let $g_{R}(r)=K_{R} / r^{\eta_{R}}$, where $K_{R}$ is a constant and $\eta_{R}$ is the path-loss exponent on the RL. In line with the fluid model, we are able to simplify equations (2), (4), (5), (6), (8) and (9):

$$
\begin{aligned}
\gamma_{0} & =\frac{(\eta-2) r^{-\eta}}{2 \pi \rho_{e N B}\left(2 R_{c}-r\right)^{2-\eta}} \\
\gamma_{i, k^{*}(i)} & =\frac{r_{i, k^{*}(i)}^{-\eta_{R}}\left(\eta_{R}-2\right)}{2 \pi \rho_{e N B}\left(2 R_{c}-r_{i, k^{*}(i)}\right)^{2-\eta_{R}}} \\
\Omega_{i} & =\frac{P_{R} K_{R}\left(2 R_{c}-r_{i, k^{*}(i)}\right)^{2-\eta_{R}}(\eta-2)}{P K\left(2 R_{c}-r\right)^{2-\eta}\left(\eta_{R}-2\right)} \\
I_{2} & =\frac{N_{t h}(\eta-2)}{2 \pi \rho_{e N B} P K\left(2 R_{c}-r\right)^{2-\eta}} \\
\Omega_{i, j} & =\frac{\left(2 R_{c}-r_{i, k^{*}(i)}\right)^{2-\eta_{R}}}{\left(2 R_{c}-r_{j, k^{*}(j)}\right)^{2-\eta_{R}}} \\
I_{3} & =\frac{N_{t h}\left(\eta_{R}-2\right)}{2 \pi \rho_{e N B} P_{R} K_{R}\left(2 R_{c}-r_{j, k^{*}(j)}\right)^{2-\eta_{R}}}
\end{aligned}
$$

Above equations, along with equations (1), (3) and (7), allow to quickly compute the SINR of a terminal in the cell of interest. The only required distances for this computation are the distance to the eNB and the distance for each $i$ to the nearest type $i$ relay. 


\section{Validation of the Fluid Model for Relay-Based Networks}

In this section, we propose a validation of the fluid model for LTE-A networks with relays presented in the last section. In this perspective, we will compare the Cumulative Distribution Function (CDF) of the SINR obtained by using fluid expressions established in section IV-B to those obtained numerically by Monte Carlo simulations. Our simulator assumes a central hexagonal cell surrounded by 10 rings of interferers. Moreover, $3 \mathrm{RNs}$ are located in each macro cell. The distance between a eNB and its associated RNs is $R_{R}$, the transmitting powers are respectively $31 \mathrm{dBm}(\mathrm{RNs})$ and $43 \mathrm{dBm}$ (eNBs). We assume a uniform distribution of UEs.

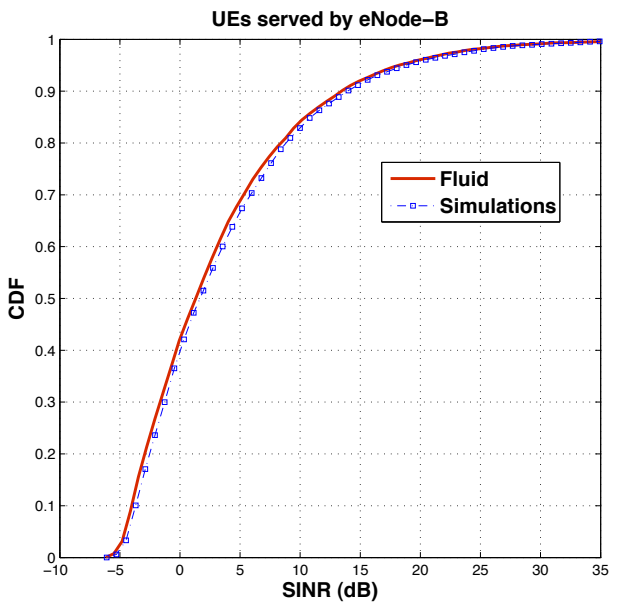

Fig. 2. CDF of the SINR for a UE connected to a eNB for $R_{R}=0.7 R_{c}$.

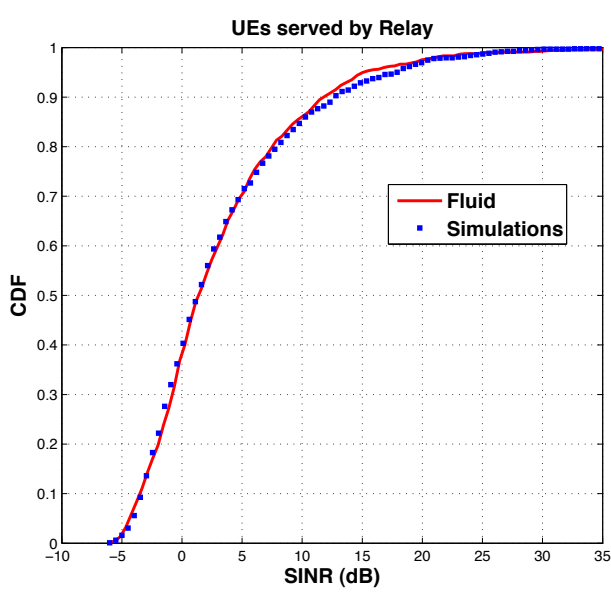

Fig. 3. CDF of the SINR for a UE connected to a RN for $R_{R}=0.7 R_{c}$.

Figures 2 and 3 show that the CDF calculated by using the fluid model for relay-based networks are very close to the ones obtained by simulations. Figure 4 shows the variation of the average UE SINR connected to a relay with respect to the distance to this relay. It can be observed that the SINR established by simulations are the same than the ones

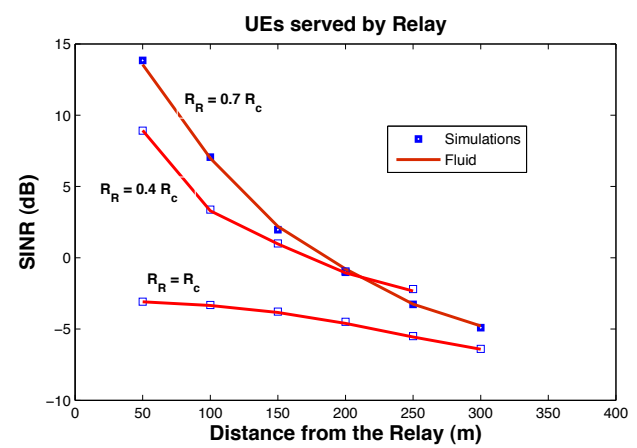

Fig. 4. Average SINR for a UE connected to a RN for $R_{R}=0.4 R_{c}$, $R_{R}=0.7 R_{c}$ and $R_{R}=R_{c}$

calculated by the fluid model. The SINR is a decreasing function of $R_{R} / R_{c}$ because useful received power decreases, and interference increases, with the distance to the serving station.

\section{Relay Enhanced Cellular Network PERFORMANCE}

In this section, we provide some performance results assuming the following set of parameters [10]: $\eta=\eta_{R}=3.41$, $K=K_{R}=5 e-4, R=1 \mathrm{Km}, N_{t h}=-104 \mathrm{dBm}$, $W=10 \mathrm{MHz}$ and $P=43 \mathrm{dBm}$. The throughput achievable at distance $r$ is modeled as follows [11]:

$$
c(r)= \begin{cases}0 & \text { if } \gamma<-10 \mathrm{~dB} \\ 0.6 W \log _{2}(1+\gamma(r)) & \text { if }-10 \leq \gamma \leq 22 \mathrm{~dB} \\ 4.4 & \text { if } \gamma>22 \mathrm{~dB}\end{cases}
$$

where $W$ is the system bandwidth.

\section{A. Cumulative Density Function of the SINR}

Fig. 5 shows the impact of the locations of relays on the CDF of the SINR when 3 RNs are deployed in the cell. We observe a small degradation of the radio quality. Indeed, as the number of interfering stations increases with the presence of $3 \mathrm{RNs}$ in the cell, and as we supposed that RNs and eNBs transmit simultaneously, UEs experience a higher interference power. For example, without relays, about $20 \%$ of the UEs have a SINR lower than $0 \mathrm{~dB}$, while with relays located at $R_{R}=0.2 R_{c}$, about $35 \%$ of UEs have a SINR lower than $0 \mathrm{~dB}$. It is very likely that while locally, some UEs see their SINR improved when relays are deployed, we observe a global degradation of the SINR.

\section{B. Cell Spectral Efficiency}

We define the cell spectral efficiency as follows:

$$
C_{\text {cell }}=(1-\tau)\left(C_{e N B}+\sum_{i=1}^{n} C_{R N i}\right),
$$

where $C_{e N B}$ and $C_{R N i}$ are the spatial averages of the throughput $c(r)$ over the surface controlled by the eNB and RN of type $i$ resp. Fig. 6 (left) shows how $C_{\text {cell }}$ is increasing with the number of RNs when $\tau=0$. This is indeed due to the 


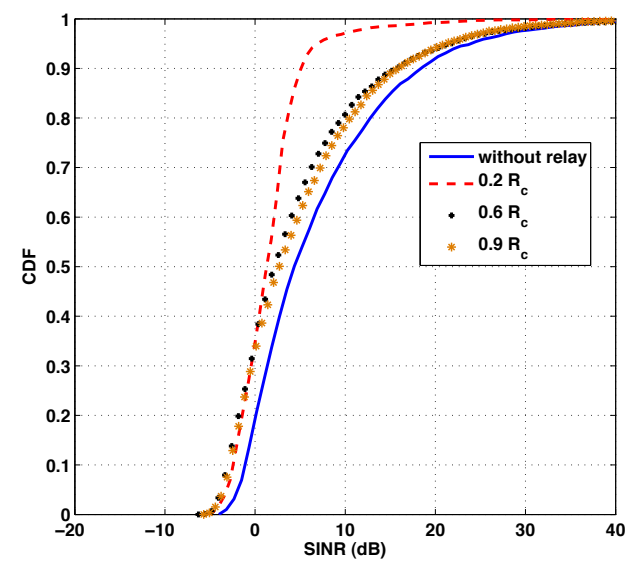

Fig. 5. CDF of the SINR with 3 RNs deployed in the cell, as a function of the distance between eNBs and RNs.

fact that, within a cell, several UEs are simultaneously served (by the eNB and the RNs). When $\tau=0$, relay deployment is equivalent to a network densification, which is known to increase the network capacity (see [12]). Fig. 6 has been obtained by optimizing the distance $R_{R}$ for every considered number of RNs $n$ (exhaustive search has been performed between $0.1 R_{c}$ and $R_{c}$ by steps of $0.1 R_{c}$ ). Let notice that the capacity is calculated instantaneously, by using the fluid model expressions, for different relays locations.

We now consider the loss due to the transmission on the BL, while taking into account parameter $\tau$ (constant). Fig. 6 (right) gives the threshold value $\tau^{*}(n)$ below which it is interesting to deploy relays. As an illustrative example, it is worth deploying four relays provided that the BL does not consume more than $80 \%$ of the radio resources. If $\tau<\tau^{*}(n)$, deploying $n$ relays results in a capacity increase. For example for $n=3$ relays, $\tau$ can reach up to $70 \%$ of the frame without capacity degradation. We see that, thanks to densification, adding more relays increases cell capacity and allows a greater part of the frame to be dedicated to the backhaul link.

\section{ACKNOWLEDGMENT}

This work was performed thanks to the France ANR NetLearn project.

\section{CONCLUSION}

In this paper, we have studied the impact of relays on the performance of a LTE-A network, while taking into account the whole interference created by the network. We have developed and validated a new framework, the fluid model of cellular networks with relays. The simple formulas provided by this model allow a quick analysis of the SINR distribution and cell capacity. We have shown that adding relays in a LTE-A network tends to degrade the overall signal quality. Moreover, we have established an upper bound on the proportion of radio resources dedicated to the backhaul link, above which it is not convenient to deploy relays. Below this
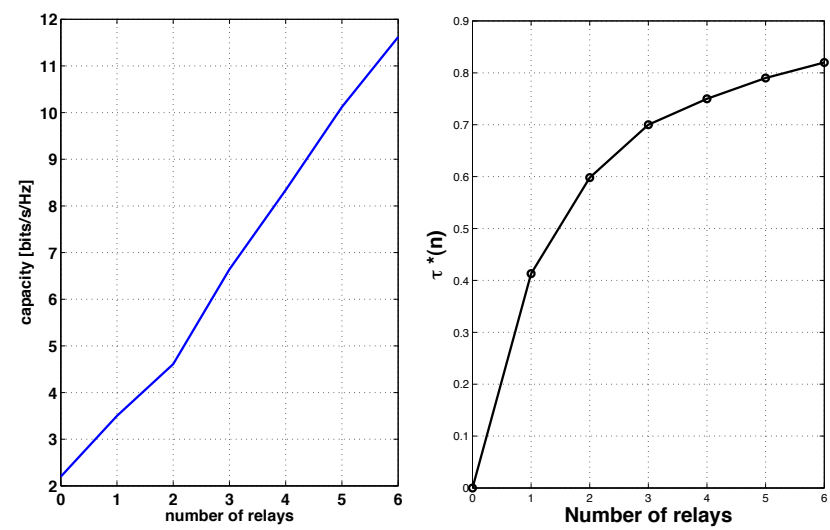

Fig. 6. Spectral efficiency per cell for $\tau=0$ (left) and threshold value for $\tau$ (right) as a function of the number of relays $\left(\tau^{*}(n)\right)$.

bound, the gain achieved by network densification outperforms the observed losses. Our further work includes the study of shadowing impact in this context. At last, we intend to study inhomogeneous relay deployments across adjacent cells.

\section{REFERENCES}

[1] Kyungmi Park, C.G. Kang, Daeyoung Chang, Seungho Song, Jongguk Ahn, and Jongtae Ihm. Relay-enhanced cellular performance of OFDMA-TDD system for mobile wireless broadband services. In Computer Communications and Networks, 2007. ICCCN 2007. Proceedings of 16th International Conference on, pages 430-435, Aug. 2007.

[2] Nokia Siemens Networks. Type-1 Relay Performance for Downlink. Technical Report R1-106216, 3GPP, Nov. 2010.

[3] R. Combes, Z. Altman, and E. Altman. Self-organizing relays: Dimensioning, self-optimization, and learning. Network and Service Management, IEEE Transactions on, 9(4):487-500, Dec. 2012.

[4] Wern-Ho Sheen, Shiang-Jiun Lin, and Chia-Chi Huang. Downlink optimization and performance of relay-assisted cellular networks in multicell environments. Vehicular Technology, IEEE Transactions on, 59(5):2529-2542, Jun. 2010.

[5] W. Guo and T. O'Farrell. Relay deployment in cellular networks: Planning and optimization. Selected Areas in Communications, IEEE Journal on, PP(99):1 -10, Oct. 2012.

[6] A.B. Saleh, O. Bulakci, J. Hämäläinen, S. Redana, and B. Raaf. Analysis of the impact of site planning on the performance of relay deployments. Vehicular Technology, IEEE Transactions on, 61(7):3139-3150, Sep. 2012.

[7] Z. Zhou, A.J. Goldsmith, and Z. Niu. On Optimal Relay Placement and Sleep Control to Improve Energy Efficiency in Cellular Networks. In Proc. of IEEE Int. Conf. on Communications (ICC), June 2011.

[8] J.-M. Kelif and E. Altman. Downlink Fluid Model of CDMA Networks. In Proc. of IEEE Vehicular Technology Conference (VTC Spring), MayJune 2005.

[9] J.-M. Kelif, M. Coupechoux, and P. Godlewski. A Fluid Model for Performance Analysis in Cellular Networks. EURASIP Journal on Wireless Communications and Networking, 2010, Article ID 435189, 2010.

[10] 3GPP. TR 25.816 v7.0.0 - Technical Specification Group Radio Access Network: UMTS 900 MHz Work Item Technical Report (Release 7) (2005-12), Dec. 2005.

[11] J. Ellenbeck, J. Schmidt, U. Korger, and C. Hartmann. A Concept for Efficient System-Level Simulations of OFDMA Systems with Proportional Fair Fast Scheduling. In Proc. of IEEE GLOBECOM Workshops, Dec. 2009.

[12] J. M. Kélif and M. Coupechoux. Cell Breathing, Sectorization and Densification in Cellular Networks. In Proc. of Int. Symposium on Modeling and Optimization in Mobile, Ad Hoc, and Wireless Networks (WiOpt), June 2009. 